\title{
Video Article \\ Human Fear Conditioning Conducted in Full Immersion 3-Dimensional Virtual Reality
}

\author{
Nicole C. Huff ${ }^{1}$, David J. Zielinski ${ }^{2}$, Matthew E. Fecteau ${ }^{1}$, Rachael Brady ${ }^{2}$, Kevin S. LaBar ${ }^{1}$ \\ ${ }^{1}$ Center for Cognitive Neuroscience, Duke University \\ ${ }^{2}$ Pratt School of Engineering, Duke University
}

Correspondence to: Kevin S. LaBar at klabar@duke.edu

URL: https://www.jove.com/video/1993

DOI: doi:10.3791/1993

Keywords: JoVE Neuroscience, Issue 42, fear conditioning, virtual reality, human memory, skin conductance response, context learning

Date Published: 8/9/2010

Citation: Huff, N.C., Zielinski, D.J., Fecteau, M.E., Brady, R., LaBar, K.S. Human Fear Conditioning Conducted in Full Immersion 3-Dimensional Virtual Reality. J. Vis. Exp. (42), e1993, doi:10.3791/1993 (2010).

\section{Abstract}

Fear conditioning is a widely used paradigm in non-human animal research to investigate the neural mechanisms underlying fear and anxiety. A major challenge in conducting conditioning studies in humans is the ability to strongly manipulate or simulate the environmental contexts that are associated with conditioned emotional behaviors. In this regard, virtual reality (VR) technology is a promising tool. Yet, adapting this technology to meet experimental constraints requires special accommodations. Here we address the methodological issues involved when conducting fear conditioning in a fully immersive 6-sided VR environment and present fear conditioning data.

In the real world, traumatic events occur in complex environments that are made up of many cues, engaging all of our sensory modalities. For example, cues that form the environmental configuration include not only visual elements, but aural, olfactory, and even tactile. In rodent studies of fear conditioning animals are fully immersed in a context that is rich with novel visual, tactile and olfactory cues. However, standard laboratory tests of fear conditioning in humans are typically conducted in a nondescript room in front of a flat or 2D computer screen and do not replicate the complexity of real world experiences. On the other hand, a major limitation of clinical studies aimed at reducing (extinguishing) fear and preventing relapse in anxiety disorders is that treatment occurs after participants have acquired a fear in an uncontrolled and largely unknown context. Thus the experimenters are left without information about the duration of exposure, the true nature of the stimulus, and associated background cues in the environment ${ }^{1}$. In the absence of this information it can be difficult to truly extinguish a fear that is both cue and contextdependent. Virtual reality environments address these issues by providing the complexity of the real world, and at the same time allowing experimenters to constrain fear conditioning and extinction parameters to yield empirical data that can suggest better treatment options and/or analyze mechanistic hypotheses.

In order to test the hypothesis that fear conditioning may be richly encoded and context specific when conducted in a fully immersive environment, we developed distinct virtual reality 3-D contexts in which participants experienced fear conditioning to virtual snakes or spiders. Auditory cues co-occurred with the CS in order to further evoke orienting responses and a feeling of "presence" in subjects ${ }^{2}$. Skin conductance response served as the dependent measure of fear acquisition, memory retention and extinction.

\section{Video Link}

The video component of this article can be found at https://www.jove.com/video/1993/

Protocol

\section{A. Stimuli and Task Design}

\section{General Design.}

We examined contextual influences on fear acquisition and memory retention over two days. This design is in parallel with rodent studies that account for neurobiological consolidation processes of long-term memory formation ${ }^{3}$ and real world contingencies in which fear is learned at a temporal distance from therapy and re-exposure experiences. Dynamic conditioned stimuli (CS) (moving snakes and spiders) were encountered in a fully immersive virtual environment known as Duke's Immersive Virtual Environment (DiVE), and were conditionally paired with the presentation of electrical wrist stimulation. A differential fear conditioning procedure was employed using skin conductance response (SCR) as a dependent measure of fear. Here we demonstrate conditioned fear and subsequent memory retention that was tested over two days in the DiVE in 26 healthy male and female participants, ages 18-30 yrs old at Duke University. This protocol was approved and in accordance with Duke University IRB standards. 


\section{Participant set up in DiVE.}

The DiVE is a fully enclosed, six-sided, $3 \mathrm{~m} \times 3 \mathrm{~m} \times 3 \mathrm{~m}$, back-projected virtual reality (VR) environment. The DiVE is located in a specially constructed 30ft cube (Control Room (VisRoom), Figure 1) in the Center for Interdisciplinary Engineering, Medicine and Applied Science at Duke University. Fear conditioning in the DiVE was conducted as described above.

Participants were seated in the center of the DiVE facing forward with head tracking on the 3-D eye glasses. Participants are taken on a fixed "virtual walk through the designated environment during each learning phase where the virtual snakes and spiders are encountered. These postural constraints were made to avoid dizziness, account for variability in height, control for amount of context and stimulus exposure between participants, and to ensure that the visual display is realistically updated according the participants' movement through the scenario.

\section{Discrimination Conditioning Procedure.}

A discrimination procedure was employed, in which the presentation of one visual CS is partially reinforced (40\% reinforcement rate) by a coterminating electrical stimulus, the unconditioned stimulus (US) during the acquisition phase. Participants were assigned to one of 2 conditions: fear acquisition to virtual snakes or to virtual spiders. The reinforced stimuli paired with the US are referred to as "CS+ while the other visual stimulus "CS-" is explicitly unpaired as a control. The CS+ and CS- were randomly assigned and counterbalanced across groups.

\section{Conditioned Stimuli.}

The stimuli were dynamic snakes and spiders that individually appear in the middle and center of the front screen of the DiVE for a duration of 4 sec. This co-occurred with an auditory stimulus signaling the appearance of a snake or spider to alert the participant to the presence of a novel stimulus in the environment (rattle or tapping sound, respectively). The virtual scene along with the snakes ad spiders were created using Maya animation software and imported into the Virtools software (Virtool SA, The Behavior Company, Paris, France) for viewing in the DiVE.

\section{Unconditioned Stimuli.}

Electrical stimulation was adjusted prior to the start of the experiment according to each subject's tolerance level in order to facilitate group comparisons and eliminate confounding influences of overall arousal level differences across groups ${ }^{4,5}$. The stimulation level was chosen by each participant to be his or her perception of "highly annoying but not painful using an ascending staircase procedure. Voltage was initially set at a low level of $30 \mathrm{~V}$ and increased in increments of $5 \mathrm{~V}$ until participants indicated that their tolerance level had been reached without inducing pain. Stimulation $(200 \mathrm{msec}$ duration delivered at $30-50 \mathrm{~Hz}$ ) was administered transcutaneously over the median nerve of the participants' dominant wrist by a bipolar surface-stimulating electrode (21 mm electrode spacing: Grass-Telefactor Model F-E 10S2, West Warwick, RI). The electrode leads were secured by a rubber strap and were attached to a Grass-Telefactor SD-9 stimulator via coaxial cable leads that were shielded and grounded through a radiofrequency filter. A saline-based gel (Sigma Gel: Parker Laboratories, Fairfield, NJ) was used as an electrolyte conductor (see Figure 2). Participants were told that all pulses would be delivered at the same intensity.

\section{Training Phases.}

The experiment described here was conducted in two sessions with a 24 hour delay. During the first session, the initial habituation period consisted of 4 trials of each CS type viewed in a grey background in 3-D full immersion but presented without reinforcement or the virtual world in which training or testing occurred. This phase allowed for acclimation to the experimental environment in the DiVE and reduction of orienting responses to the conditioned stimuli. Immediately after the habituation phase, the fear acquisition phase consisted of 16 intermixed trials of each CS type, in which the CS- is presented alone and 5 of the 16 CS+ trials are reinforced. Approximately 24 hrs later, testing for memory retention and the extinction training occurred. This phase consisted of 16 trials of each CS type with no US, in a virtual context that was either the same as or different from the fear acquisition context (counterbalanced across participants). One context was an indoor environment (interior of a furnished apartment, Context A) and the other context was an outdoor environment (neighborhood scene, Context B). Subjects were randomly assigned to an experimental group, which determined the order of context presentation on Days 1 and 2 . They were either assigned to the Same Context condition (AA or BB) or a Context Shift condition (AB or BA). The path length and course were matched for consistency between virtual worlds, as were the number and placement of objects/stimuli within the different environments.

\section{Experimental Parameters.}

The inter-trial interval was $14 \pm 2 \mathrm{sec}$. The sequence of CSs was pseudorandom, subject to the constraint that no more than 2 trials of the same CS occur consecutively (to avoid confounding inductions of state anxiety and cognitive expectancy). Partial reinforcement (40\%) of the CS+ was used to delay rapid extinction that normally occurs in human participants following $100 \% \mathrm{CS}+$ reinforcement $^{6,7}$. In addition, partial reinforcement provides a more realistic conditioning contingency to the extent that aversive events do not always occur following a feared stimulus.

\section{Task Instructions.}

Prior to each experimental phase, participants were informed about the following design features: they would encounter animated snakes and spiders in the virtual environment; they would be guided through the environment in a virtual walk along a fixed path; and they may receive electrical stimulation on the wrist at the level that was set prior to conditioning at any time throughout the study. They were instructed to face directly forward and attend to snake and spiders images presented in the center of the front screen, and reminded that they did not have any control over their own movement through the world or the occurrence of electrical stimulation. They were also informed that they could terminate the study at any time without penalty to them. 


\section{B. Psychophysiological Measurements}

\section{Data Collection.}

SCR was used as the dependent measure of fear, as described previously ${ }^{4,6}$. SCR was recorded via a psychophysiological monitoring system (BIOPAC Systems, Santa Barbara, CA). SCR was monitored from silver-silver chloride electrode disks attached by Velcro straps to the middle phalanges of the 1st and 2nd digits of the non-dominant hand. A saline-based gel (Sigma Gel) was used as a conductive electrolyte. Subjects were instructed to keep their hand still to avoid movement artifacts in the SCR recording electrode. Leads reached the BIOPAC physiological recording system which is located just outside the DiVE in the control room. The BIOPAC system synchronizes with the stimulus presentation computer running Virtools software. Figure 1 illustrates a participant in the DiVE, immersed in Context A. The technical set up of the control computer (Virtools and script generation), BIOPAC (SCR), and electrical stimulator are illustrated in Figure 2.

Skin conductance was sampled at $200 \mathrm{~Hz}$, amplified, and stored for offline analysis using AcqKnowledge software (BIOPAC Systems, Santa Barbara, CA). Virtools software controls the stimulus presentation and triggers the shock generator via a National Instruments DIO-24 data acquisition card (Austin, TX). The recorded waveforms are lowpass filtered using a Blackman window (cutoff frequency $=31 \mathrm{~Hz}$ ) and smoothed over 3 successive data points. Skin conductance response amplitudes were time-locked to the onset of each CS and US relative to the prestimulus baseline to derive a dependent measure of conditioned and unconditioned fear, respectively ${ }^{4-6,8}$. For inclusion in the data analysis, the following criteria were established: latency $=1-4 \mathrm{~s}$, duration $=0.5-5 \mathrm{~s}$, and minimum amplitude $=0.02$ micro Siemens ( $\mu \mathrm{S}$ ). Responses that do not meet these criteria are scored as zero.

\section{Analysis of SCR.}

Because SCR data is typically skewed toward zero, the data were square-root transformed prior to statistical analysis to attain a normal distribution. The data from each CS type (virtual snakes or spiders) were collapsed into 'early' and 'late' trial blocks of each phase, as learning typically varies across time within each learning phase. Repeated Measures Analyses of Variance (ANOVA) were used to compute group differences in conditioned skin conductance responses as a function of learning Phase and CS Type as within subjects variables (Late Acquisition (CS+, CS-), Early or Late Extinction (CS+, CS-) and Context assignment (Same or Shift) as the between-subjects variable. Data were normalized by dividing the conditioned response values on each trial by each participants' own maximum US response to wrist stimulation (on any trial) to account for individual variations in responding and to rule out non-responders (individuals who show little or no SCRs). For data visualization in Figure 3 differential SCR scores were calculated as an index of learning by subtracting responses to the CS- from those of CS+ across trial blocks. According to this measure, difference scores of zero reflect no learning, whereas difference scores above zero reflect learning of a fear response. However, to statistically determine retention of contextual fear as shown in Figure 3 a Student's t-test was computed on SCR values to the CS+ and CS- at Early Extinction on Day 2 as a function of the context manipulation (Same Context vs. Context Shift as a betweengroups analysis).

\section{Hardware System Description}

The Duke University DiVE system is based on the projected virtual reality "CAVE" design ${ }^{9}$. The DiVE system is $3 \mathrm{~m} \times 3 \mathrm{~m} \times 3 \mathrm{~m}$ room where all 6 "walls" ( 4 walls, the ceiling and the floor) show stereographic computer images by rear projection. Each wall has a DLP projector (Christie Digital Mirage S+2K, operating at $1056 \times 1056 @ 110 \mathrm{hz}{ }^{10}$ ) which in turn is controlled by a dedicated render computer (Windows XP dual core 2.0 $\mathrm{GHz}$ with nVidia Quadro 3000FX-G graphics cards). One wall slides open to allow access into and out of the DiVE.

The 6 render computers are controlled by a master computer that communicates to the tracking system (Intersense IS-900 ${ }^{11}$ ), controls the sound system, and sends a pulse through the parallel port to the electrical shock system. The tracking system provides 3D location and orientation information for the participant's head and hand positions. Active stereographic vision is provided through liquid crystal shutter glasses $\left(\right.$ CrystalEyes $3^{12}$ ). The seven computers ( 6 render computers and master computer) are synchronized on image frame boundaries through the genlock (G-sync) capability of the nVidia graphics cards.

\section{Software Description}

The fear conditioning and retention testing contexts for this experiment consists of two different virtual worlds through which participants are taken on a guided tour. The virtual worlds were modeled using the 3D modeling package Maya ${ }^{13}$. Navigation is limited to a fixed path that is identical in all virtual worlds. Movement along this path is controlled through the Virtools ${ }^{14}$ software system. Virtools is a game engine designed primarily for a desktop or web-based experience. Through the VRPack extension of Virtools, virtual worlds are projected in the DiVE.

Virtools communicates with the tracking system through the Virtual Reality Peripheral Network (VRPN ${ }^{15}$ ), an open source library. VRPN registers the participants head and hand location and orientation as well as button press information. Virtools uses the head tracking information to render the $3 \mathrm{D}$ scene at the correct perspective for the participant. 


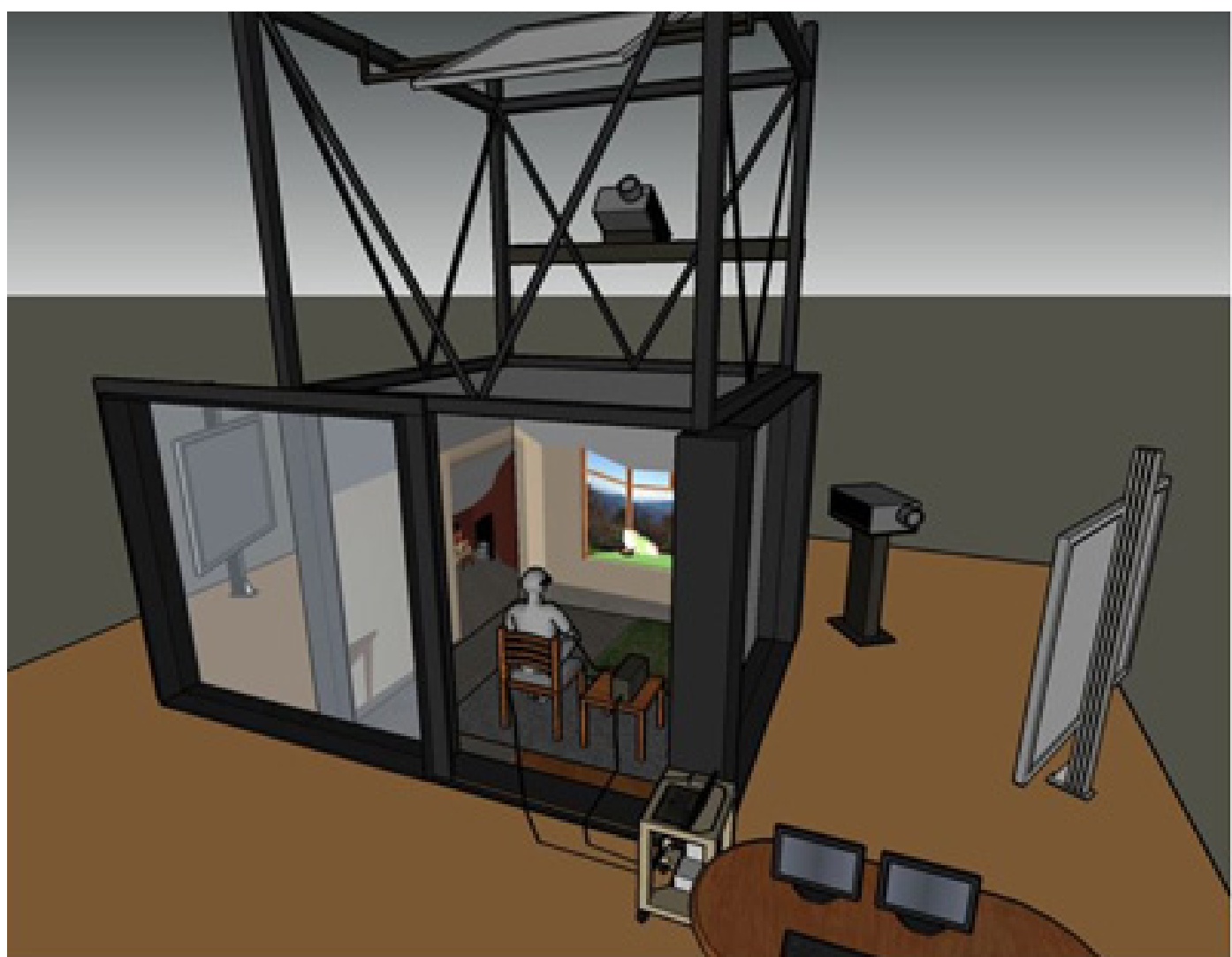

Figure 1. Schematic of the control room (VisRoom) and the DiVE cube with a human participant viewing a virtual scene.

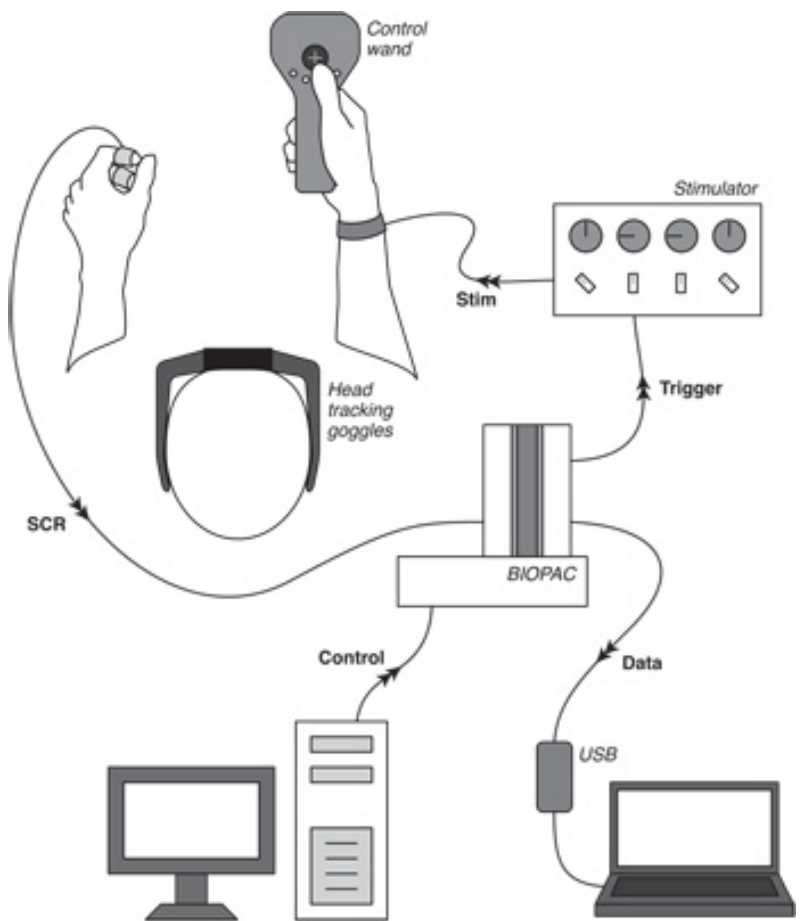

Figure 2. Diagram of a participant with skin conductance electrodes on left hand measuring tonic and phasic responses to stimuli. Electric stimulator electrodes are on right wrist. BIOPAC collects physiological data via Acknowledge software on laptop computer. Codes are sent via OSC from desktop computer where Virtools software generates virtual reality scripts projected in the DiVE. 


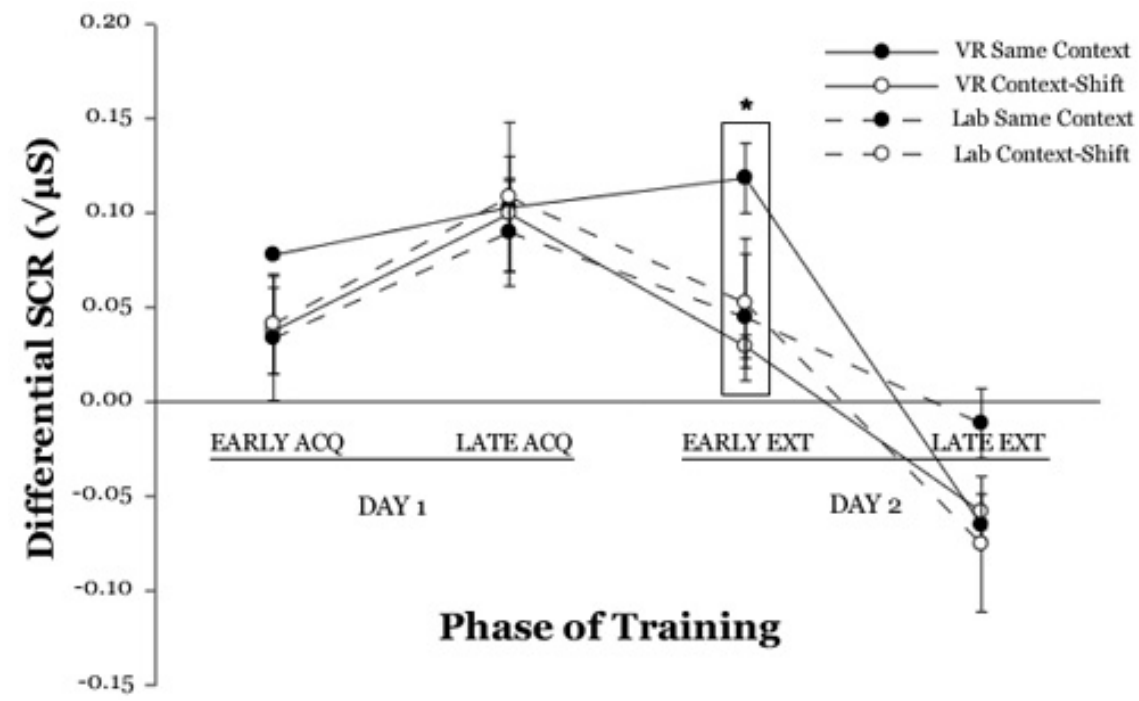

Figure 3. Comparison of Fear Acquisition and Extinction in DiVE and Laboratory. Differential Skin Conductance Response (SCR) +/- SEM in participants conditioned and retested 24 hrs later in the Laboratory or Virtual Reality (DiVE). Graphic illustrates equivalent fear acquisition and extinction in participants in the DiVE and laboratory studies. Same Context $(n=12)$ testing on Day 2 in DiVE yields more robust fear memory retention relative to Shifted Context $(n=14)$ measured by SCR to CS+, in our DiVE participants but not in our laboratory participants, * $p=.05$.

\section{Discussion}

\section{Results}

Equivalent within-session fear acquisition and extinction across groups was found (Figure 3). These data indicate that reliable and informative fear conditioning studies can be performed within the constraints and capabilities of a fully immersive environment. Moreover we also demonstrate robust contextual fear memory in the Same Context fear retention participants in the DiVE (participants who remained in the same context for Days 1 and 2, relative to those who experienced a context shift). The retention of fear is stronger in the DiVE than that observed in a conventional laboratory matched paradigm ${ }^{16}$ (see Figure 3). With the immersive VR setup, one can also examine and manipulate rich contextual environments to probe declarative memory processes in humans, unlike in the laboratory setting where realistic multimodel context manipulations are difficult to accomplish. Finally, the VR worlds can easily be ported for use in conjunction with functional magnetic resonance imaging (fMRI) using stereoscopic VR goggles to conduct brain activation analysis during encoding or retrieval of fear acquisition, extinction, and relapse. This methodology can be utilized to bridge rodent and clinical findings in fear and anxiety.

\section{Controlling context and stimulus exposure in virtual reality.}

A major issue with exploiting VR for experimental use is also its strength. Specifically, fully immersive VR provides the complexity, confounds, and freedom of the real world. For example, in real life, trauma victims experience an aversive stimulus in a context for an unknown amount of time. The contextual exposure, specific features and other sensory input that were attended are also unknown, or not confirmable. By the same token, if we were to allow participants to freely explore the virtual environments we would not be able to account for context or stimulus exposure time or duration. For example, one participant may walk very quickly, and miss 3 out of $4 \mathrm{CS}+$ presentations. Another may explore only one room in the virtual apartment. Likewise, if stimulus presentation is not specified in the center of the screen, where gaze is directed prior to starting, participants will avoid or miss CS presentations. Our solution to these potential confounds was to take participants on a seated, guided tour of each environment at a rate that would allow for a specific interstimulus interval (ISI) and stimulus duration. We could then extract comparable SCR data from specific time points and specific locations across all participants (e.g., responses to the CS+, US, and CS- stimuli). Difficulties encountered after making this decision included finding a path shape, length, and movement rate that would not cause nausea or proprioceptive dissonance to the participant, and yet feel appropriate to mimic natural ambulation through a novel environment.

\section{Implementing standard fear conditioning parameters to a VR system.}

To simulate realistic conditioned stimuli snakes and spiders were designed after wild-life images. The snakes and spiders were first modeled in Maya, a computer graphics 3D modeling and animation software package and then imported into the VR system. We did this because Virtools is a virtual reality authoring system, not a modeling application. It is therefore best used to run a VR system and add the interaction and navigation to a scene. Specifically, in Maya four different animations for each CS type were created (e.g., a coiled snake, a spider running across the floor, a snake lunging forward with open mouth) and then imported into Virtools.

Prior to importing the dynamic snake and spider models into Virtools from Maya, a path was created in Virtools to guide the participant around the environment in a smooth circular fashion so as to allow sampling of the environment over the course of 32 conditioned stimulus presentations during fear conditioning. The shape of the path is the same for each of our three virtual worlds. The path was created to stop for four seconds for each stimulus presentation, the interstimulus interval was $11+/-4$ seconds during which the subject was slowly moving (being guided) through the environment. This interval was determined from our previous fear conditioning experiments in the laboratory ${ }^{8,16}$ because it allows for recovery of the skin conductance response between stimulus presentations. Stimuli were then placed on the path at points specified by the timing parameters. This setup created specific stimulus and context conjunctions (e.g., a snake slithering on the dining room table, a spider walking around the sofa leg), which can later be probed for explicit memory. Stimuli appearances were pseudo-randomized through the scripts. 
All stimulus presentations appeared in the middle of the front screen to prevent the participant from having to search for the stimulus. This provided us with a controlled amount of stimulus exposure time, and a defined context location. One limitation of the forward view is that it does not take advantage of the full capabilities of the immersive system (e.g., snakes cannot enter the room from behind the participant). Additionally, stimuli were carefully placed outside of a boundary box around the participants location so that the snakes and spiders never encroached upon the participants personal space.

\section{View point and head tracking.}

The angle of the DiVE was set so that from a seated position the participant had a correct forward facing angle. This controlled for variations in height between participants, and minimized movement artifacts on our physiological recordings. Participants were instructed to face forward and move as little as possible, this also controlled for where participants were looking, and therefore maintained consistent stimulus and context exposure between participants. We chose to turn on a head-tracking device in the 3D goggles worn by the participants to ensure they were viewing the scene with the correct perspective. If head tracking had not been elected for, head movement to the left or right would not correctly occlude how the objects appeared in the world (e.g. objects would appear bent on screens in the DiVE as participants walked through). With head tracking elected for, we could be sure that features in the environment retained their normal proportions and were drawn correctly on each of the six walls of the DiVE for the duration of the experiment.

\section{Data Collection.}

In our standard laboratory version of fear conditioning ${ }^{8,16}$ stimulus presentation was controlled by computer script programmed in the Presentation software package. In order to maintain consistency between the lab and the virtual environment, we imported our standard fear acquisition and extinction scripts in code format to the control computer in the control room that hosts the DiVE cube (see Figure 1). The parallel port code was set to send a generated list of numeric codes to signal distinct events, such as presentations of the snake, spider, and electrical stimulation onsets. In our design, Virtools sends an Open Sound Control message (OSC ${ }^{17}$ ) OSC/UDP message to a custom C++ program that sets the parallel port value. Our C++ program uses the OSCpack ${ }^{18}$ library.

The BIOPAC's digital input is connected to the computer parallel port. SCR data is collected on the laptop computer from BIOPAC via parallel port, then normalized, and calculated to $\mathrm{CS}+$ / CS- and US onsets within specific parameters (see above for details). In addition to rendering the scene and controlling navigation, Virtools is also used to log user events (button presses). In summary, during an experiment, messages are sent from the master computer to the BIOPAC system through the parallel port. Because Virtools cannot communicate with the parallel port on the computer directly a small $\mathrm{C}++$ program listens for an OSC message from Virtools and then transmits it to the parallel port.

\section{Disclosures}

No conflicts of interest declared.

\section{Acknowledgements}

We thank Holton Thompson for her work in creating the Virtools 3-D worlds in Maya and Eric Monson for the schematic drawings. Research was sponsored in part by postdoctoral NIH F32 MH078471 to N.C.H, and NIDA RO1 DA027802 to K.S.L.. The DiVE was funded by NSF BCS-0420632.

\section{References}

1. Mineka, S. \& Zinbarg, R. A contemporary learning theory perspective on the etiology of anxiety disorders: It's not what you thought it was. Amer. Psychol. 61, 10-26, (2006).

2. Sanchez-Vives, M.V. \& Slater, M. From presence to consciousness through virtual reality. Nat. Rev. Neurosci. 6, 332-9, (2005).

3. McGaugh, J.L. Memory--- a century of consolidation. Science. 287, 248-51, (2000).

4. LaBar, K.S., Cook, C.A., Torpey, D.C., \& Welsh-Bohmer, K.A. Impact of healthy aging on awareness and fear conditioning. Behav Neurosci.118, 905-15, (2004).

5. LaBar, K.S. \& Phelps, E.A. Reinstatement of conditioned fear in humans in context dependent and impaired in amnesia. Behav Neurosci. 119, 677-86, (2005).

6. LaBar, K.S., Gatenby, J.C., Gore, J.C., LeDoux, J.E., \& Phelps, E.A. Human amygdala activation during conditioned fear acquisition and extinctions: a mixed trial fMRI study. Neuron. 20,937-457, (1998).

7. Phelps, E.A., Delgado, M.R., Nearing, K.I., \& LeDoux, J.E. Extinction learning in humans: role of the amygdala and mPFC. Neuron. 43 , 897-905, (2004).

8. Zorawski, M., Cook, C.A., Kuhn, C.M., \& LaBar, K.S. Sex, stress, and fear: individual differences in conditioned learning. Cogn. Affect. Behav. Neurosci. 5, 191-201, (2005).

9. Cruz-Neira, C., Sandin, D., \& DeFanti, T. Surround-screen projection-based virtual reality: The design and implementation of the CAVE. Acm. Siggraph. Proc. 93,135-142, (1993).

10. Christie: Mirage 2000 - SXGA 2000 Lumen Active Stereoscopic Projector [Internet]. Cypress (CA): Christie Digital Systems USA, Inc. [cited 2010 June 17]. Available from: http://www.christiedigital.com/AMEN/Products/mirage200038DMD02312.

11. Intersense: Sensing Every Move [Internet]. Billerica (MD): InterSense, Inc.; IS-900 Systems [cited 2010 June 17]. Available from: http:// www.intersense.com/IS-900_Systems.aspx.

12. RealD: The New 3D [Internet]. Beverly Hills (CA): RealD; CrystalEyes 3 [cited 2010 June 17]. Available from: http://www.reald.com/Content/ contact.aspx.

13. Wikipedia: The Free Encyclopedia [modified 2010 June 13]. San Francisco (CA): Wikimedia Foundation, Inc.; Autodesk Maya [cited 2010 June 17]. Available from: http://en.wikipedia.org/wiki/Autodesk_Maya. 
14. Dassault Systemes [Internet]. Velizy-Vilacoublay, France: Dassault Systemes.; 3DVIA Virtools [cited 2010 June 17]. Available from: http:// a2.media.3ds.com/products/3dvia/3dvia-virtools/.

15. VRPN 07.26: Virtual Reality Peripheral Network [Internet]. Chapel Hill (NC): University of North Carolina at Chapel Hill, Department of Computer Science; [Internet]. VRPN 07.26; [cited 2010 June 17]. Available from: http://www.cs.unc.edu/Research/vrpn/index.html.

16. Huff, N.C., Hernandez, J.A., Blanding, N.Q., \& LaBar, K.S. Delayed extinction attenuates fear renewal and spontaneous recovery in humans. Behav. Neurosci. 123, 834-43, (2009).

17. Wikipedia: The Free Encyclopedia [modified 2010 June 9]. San Francisco (CA): Wikimedia Foundation, Inc.; Open Sound Control [cited 2010 June 17]. Available from: http://en.wikipedia.org/wiki/Open_Sound_Control.

18. Oscpack: A simple C++ Packet Manipulation Library [Internet]. [place unknown]: oscpack [cited 2010 June 17]. Available from http:// www.audiomulch.com/ rossb/code/oscpack/. 